\title{
Erratum to: Modeling and predicting drug resistance rate and strength
}

\author{
R. Fullybright ${ }^{1} \cdot$ A. Dwivedi ${ }^{2} \cdot$ I. Mallawaarachchi ${ }^{2} \cdot$ B. Sinsin $^{3}$
}

Published online: 26 August 2016

(C) Springer-Verlag Berlin Heidelberg 2016

Erratum to: Eur J Clin Microbiol Infect Dis (2016) 35:1259-1267

DOI: 10.1007/s10096-016-2659-z

Electronic supplementary materials (ESM) $1-10$ of the originally published article were incorrect. The correct ESM files are published here.

The online version of the original article can be found at http://dx.doi. org/10.1007/s10096-016-2659-z.

Electronic supplementary material The online version of this article (doi:10.1007/s10096-016-2758-x) contains supplementary material, which is available to authorized users.

\footnotetext{
R. Fullybright

fullybright@gmail.com
}

1 Department of Applied Research, Applied-Research Center for True Development, 4016 rue Préfontaine, Montréal, Québec H1W 0A3, Canada

2 Division of Biostatistics and Epidemiology, Department of Biomedical Sciences, Texas Tech University Health Sciences Center, 4801 Alberta Avenue, El Paso, TX 79905, USA

3 Laboratory of Applied Ecology, School of Agronomic Sciences, University of Abomey-Calavi, 05 BP 1752 Cotonou, Republic of Benin 